Military Technical

College

Kobry El-Kobba

Cairo, Egypt

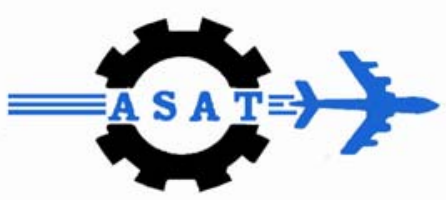

12-th International Conference

on

Aerospace Sciences \&

Aviation Technology

\title{
SIMPLIFIED VARIATIONAL APPROACH FOR ANALYSIS OF THICK ORTHOTROPIC LAMINATED
}

\section{PLATES: 2- SIMPLE SUPPORT}

Tmerek $^{*}$ M.Taha, El-Soaaly ${ }^{* *}$ E. E., El-Nomrossy ${ }^{*}$ M. M., and Istafanous ${ }^{*}$ A. A.

\begin{abstract}
A simplified variational approach, stress-based, for the analysis of symmetric crossply laminate was developed in Part 1 of this work. It was also tested for the 1-D problem, orthotropic plate in a cylindrical bending, solved exactly by Pagano. This simplified approach is extended here to a two-dimensional structure. The accuracy of the present approach is examined by applying it to the case of rectangular laminated plate with simple support for which the elasticity solution was obtained [1,2]. The present approach gives results for multi-layered laminate with small span-tothickness ratios that compare well with those from elasticity solutions and other known theories as well.
\end{abstract}

\section{KEY WORDS}

Laminate, Orthotropic, Plate, Simple Support, ESL, Variational, and Stress-based

\footnotetext{
Egyptian Armed Forced

** Dpt. of MechEng, Higher Technological Institute (HTI), Cairo, Egypt.
} 


\section{INTRODUCTION}

As the technology of composites advances, laminated composites are used for thick and moderately thick structures. Also, laminated plates made of advanced composite materials, whose elastic to shear modulus ratios are very large, are susceptible to thickness effects because their effective transverse shear moduli are significantly smaller than the effective elastic moduli.

The classical laminated plate theory, CPT, is inadequate for these types of laminates since it ignores the transverse shear effects. Thus, the shear deformation theories were emerged. Shear deformation theories, FSDT with pre-assumed continuous displacement field across the thickness require a shear correction factor and suffer from locking problem. Thus the higher order, HSDT were developed.

The continuity of the displacement field and its derivatives through the laminate thickness is in contradiction with the continuity of transverse stresses. Thus, the transverse stresses predicted are doubled value when using constitutive relations. To remove these discrepancies in ESL, the Layer Wise models, LWM were introduced which give excellent results for both global and local distributions of displacements and stress. However, LWM's suffers from a numerical crisis if the layer number becomes large.

A stress-based approach developed by the authors in a previous work [3] was tested by the bench-mark problem for the plate in a cylindrical bending solved exactly by Pagano. The presented simplified approach gave good results for out-of-plane displacement and the transverse stresses. This approach is now to be applied for the 2-D problem. Specifically, the problem of a symmetrically bidirectional laminate with pinned edges under static bending forces is considered. For the sake of brevity, the details of the derivation of equilibrium equations, variational analysis and continuity conditions are omitted, see [3]. For the sake of continuity, only relevant equations are provided.

To assess the proposed approach, the problem of bending of a 3, 5, 7 and 9-layered, symmetrically laminated rectangular and square plates simply-supported on all edges are investigated. Numerical results are given and compared with those resulting from the elasticity solution, ES, classical lamination theory, CPT, shear deformation theory (first order, FSDT and higher order, HODT) and layer wise, LWM, as well.

\section{GOVERNING EQUATIONS}

Consider a laminated plate composed of an arbitrary number of orthotropic layers such that the various axes of elastic symmetry are parallel to the plate axes. The simple support boundary conditions are expressed as

$$
\begin{array}{ll}
@ \mathrm{x}_{1}=0, \mathrm{a} ; & \sigma_{11}=\mathrm{u}_{2}=\mathrm{u}_{3}=0, \\
\text { @ } \mathrm{x}_{2}=0, \mathrm{~b} ; & \sigma_{22}=\mathrm{u}_{1}=\mathrm{u}_{3}=0
\end{array}
$$


Where $a$ and $b$ denote the length and the width of the plate, respectively. A transverse normal loading, $-\mathrm{q}_{\mathrm{o}} \sin \frac{\pi \mathrm{x}_{1}}{\mathrm{a}} \sin \frac{\pi \mathrm{x}_{2}}{\mathrm{~b}}$, is applied to the top surface, while the lower surface is traction-free.

Since the stress resultants must satisfy the following equilibrium equation[3];

$$
\begin{aligned}
& \mathrm{N}_{\alpha \beta, \beta}=0 \\
& \mathrm{M}_{\alpha \beta, \beta}-\mathrm{S}_{\alpha}=0 \\
& \mathrm{~S}_{\alpha, \alpha}+\mathrm{q}=0
\end{aligned}
$$

And using the following constitutive relations for orthotropic laminate[3]

$$
\begin{aligned}
& \mathrm{N}_{\alpha \beta}=\mathrm{h}\left(\overline{\mathrm{Q}}_{\alpha \beta \gamma \delta} \mathrm{u}_{\gamma, \delta}^{\mathrm{o}}+\overline{\mathrm{B}}_{\alpha \beta} \mathrm{p}\right) \\
& \mathrm{M}_{\alpha \beta}=\frac{\mathrm{h}^{3}}{12}\left(\overline{\mathrm{Q}}_{\alpha \beta \gamma \delta} \psi^{\gamma, \delta}+\frac{6}{5 \mathrm{~h}} \overline{\mathrm{B}}_{\alpha \beta} \mathrm{q}\right) \\
& \mathrm{S}_{\alpha}=\mathrm{h} \overline{\mathrm{d}}_{\alpha \beta}\left(\psi_{\beta}+\mathrm{w}_{, \beta}\right)
\end{aligned}
$$

where, $\alpha, \beta=1,2, \overline{\mathrm{d}}_{\alpha \beta}=5 \overline{\mathrm{C}}_{\alpha 3 \beta 3} / 6$, and $\overline{\mathrm{B}}_{\alpha \beta}=\overline{\mathrm{C}}_{\alpha \beta 33} / \overline{\mathrm{C}}_{3333}$.

The total load $\mathrm{q}$ and the mean extensional load $\mathrm{p}$ may be written as

$$
\begin{aligned}
& \mathrm{q}=-\mathrm{q}_{\mathrm{o}} \sin \frac{\pi \mathrm{x}_{1}}{\mathrm{a}} \sin \frac{\pi \mathrm{x}_{2}}{\mathrm{~b}} \\
& \mathrm{p}=-\frac{1}{2} \mathrm{q}_{\mathrm{o}} \sin \frac{\pi \mathrm{x}_{1}}{\mathrm{a}} \sin \frac{\pi \mathrm{x}_{2}}{\mathrm{~b}}
\end{aligned}
$$

The stress resultants are chosen to be in the following form

$$
\begin{aligned}
& \mathrm{S}_{1}=\mathrm{S}_{1}^{\cdot} \cos \frac{\pi \mathrm{x}_{1}}{\mathrm{a}} \sin \frac{\pi \mathrm{x}_{2}}{\mathrm{~b}}, \mathrm{~S}_{2}=\mathrm{S}_{2}^{\cdot} \sin \frac{\pi \mathrm{x}_{1}}{\mathrm{a}} \cos \frac{\pi \mathrm{x}_{2}}{\mathrm{~b}}, \\
& \mathrm{M}_{11}=\mathrm{M}_{11}^{\cdot} \sin \frac{\pi \mathrm{x}_{1}}{\mathrm{a}} \sin \frac{\pi \mathrm{x}_{2}}{\mathrm{~b}}, \mathrm{M}_{22}=\mathrm{M}_{22}^{\cdot} \sin \frac{\pi \mathrm{x}_{1}}{\mathrm{a}} \sin \frac{\pi \mathrm{x}_{2}}{\mathrm{~b}}, \mathrm{M}_{12}=\mathrm{M}_{12}^{\cdot} \cos \frac{\pi \mathrm{x}_{1}}{\mathrm{a}} \cos \frac{\pi \mathrm{x}_{2}}{\mathrm{~b}}=\mathrm{M}_{21}
\end{aligned}
$$

And the generalized displacements are also chosen as

$$
\mathrm{w}=\mathrm{w}^{\bullet} \sin \frac{\pi \mathrm{x}_{1}}{\mathrm{a}} \sin \frac{\pi \mathrm{x}_{2}}{\mathrm{~b}}, \psi_{1}=\psi_{1}^{\cdot} \cos \frac{\pi \mathrm{x}_{1}}{\mathrm{a}} \sin \frac{\pi \mathrm{x}_{2}}{\mathrm{~b}}, \psi_{2}=\psi_{2}^{\bullet} \sin \frac{\pi \mathrm{x}_{1}}{\mathrm{a}} \cos \frac{\pi \mathrm{x}_{2}}{\mathrm{~b}}
$$

Where; $\left(\mathrm{S}_{1}^{\bullet}, \mathrm{S}_{2}^{\bullet}, \mathrm{M}_{11}^{\bullet}, \mathrm{M}_{22}^{\bullet}, \mathrm{M}_{12}^{\bullet}, \psi_{1}^{\bullet}, \psi_{2}^{\bullet}\right.$ and $\left.\mathrm{w}^{\bullet}\right)$ are constants to be determined using the equilibrium equations (2), the constitutive equations (3), and satisfying boundary conditions at $\mathrm{x}_{1}=0$, a and $\mathrm{x}_{2}=0$, $\mathrm{b}$ (simple support). Since a bidirectional orthotropic laminate is assumed, we get the following 8 equations

$$
\begin{aligned}
& \mathrm{S}_{1,1}+\mathrm{S}_{2,2}=-\mathrm{q} \\
& \mathrm{M}_{11,1}+\mathrm{M}_{12,2}-\mathrm{S}_{1}=0 \\
& \mathrm{M}_{21,1}+\mathrm{M}_{22,2}-\mathrm{S}_{2}=0
\end{aligned}
$$




$$
\begin{aligned}
& \mathrm{M}_{11}=\frac{\mathrm{h}^{3}}{12}\left(\overline{\mathrm{Q}}_{1111} \psi_{1,1}+\overline{\mathrm{Q}}_{1122} \psi_{2,2}\right)+\frac{\mathrm{h}^{2}}{10} \overline{\mathrm{B}}_{11} \mathrm{q} \\
& \mathrm{M}_{22}=\frac{\mathrm{h}^{3}}{12}\left(\overline{\mathrm{Q}}_{2222} \psi_{2,2}+\overline{\mathrm{Q}}_{2211} \psi_{1,1}\right)+\frac{\mathrm{h}^{2}}{10} \overline{\mathrm{B}}_{22} \mathrm{q} \\
& \mathrm{M}_{12}=\frac{\mathrm{h}^{3}}{12} \overline{\mathrm{Q}}_{1212}\left(\psi_{1,2}+\psi_{2,1}\right)+\frac{\mathrm{h}^{2}}{10} \overline{\mathrm{B}}_{12} \mathrm{q} \\
& \mathrm{S}_{1}=\mathrm{h} \overline{\mathrm{d}}_{11}\left(\psi_{1}+\mathrm{w}_{, 1}\right) \\
& \mathrm{S}_{2}=\mathrm{h} \overline{\mathrm{d}}_{22}\left(\psi_{2}+\mathrm{w}_{, 2}\right)
\end{aligned}
$$

Solving these 8 equations leads to the determination of the both stress resultants $\left(\mathrm{S}_{1}^{*}, \mathrm{~S}_{2}^{*}, \mathrm{M}_{11}^{\cdot}, \mathrm{M}_{22}^{\cdot}, \mathrm{M}_{12}^{\cdot}\right)$ and the generalized displacements $\left(\psi_{1}^{*}, \psi_{2}^{\bullet}, \mathrm{w}^{\bullet}\right)$

\section{NUMERICAL EXAMPLE}

Here we present numerical results. The three-dimensional elasticity solutions of Pagano [1] and Pagano and Hatfield [2] for simply supported rectangular plates under sinusoidal loading are used to assess the present approach.

The following laminated plate problems are considered:

1. Square laminates consisting of 3-, 4-, 5-, 7-, and 9-layers.

2. A 3-ply laminate of rectangular geometry $(\mathrm{b} / \mathrm{a}=3)$.

All the laminates are with layers of equal thickness and subjected to sinusoidally distributed transverse loading $\mathrm{q}_{\mathrm{o}} \sin \frac{\pi \mathrm{x}_{1}}{\mathrm{a}} \sin \frac{\pi \mathrm{x}_{2}}{\mathrm{~b}}$.

Each layer is a unidireticonal fiber reinforced material with the following properties, which simulate a high modulus Graphite/Epoxy laminate

$$
\mathrm{E}_{\mathrm{L}}=172 \mathrm{GPa}, \mathrm{E}_{\mathrm{T}}=6.9 \mathrm{GPa}, \mathrm{G}_{\mathrm{LT}}=3.5 \mathrm{GPa}, \mathrm{G}_{\mathrm{TT}}=1.4 \mathrm{GPa}, v_{\mathrm{LT}}=v_{\mathrm{TT}}=0.25
$$

Where $L$ signifies the direction parallel to the fibers, $T$ is the transverse direction and $v_{\mathrm{LT}}$ is the major Poisson's ratio.

All the laminates considered are symmetric with respect the central plane, with fiber orientations alternating between $0^{\circ}$ and $90^{\circ}$ with respect to the $\mathrm{X}_{1}$-axis, and the $0^{\circ}$ layers are the outer surfaces of the laminate.

The numerical results are summarized in the following sections. Also shown, for comparison purposes, are the results given by:

1. 3-D Elasticity (exact) [4],

2. Higher order theory, HSDT, [5], [8],

3. Finite element based on Layerwise theory, LWM, [6],

4. Three-dimensional finite element [7],

5. First order shear deformation theory, FSDT, [8].

which almost represent all the models used for the analysis of laminated plates. The analogous CPT results, quoted by [1] and [2], are given as well. Although equivalent 
single layer approach is adopted, a computer program, MathCad, was necessary to find the expressions of the eight unknowns, see Appendix.

Max Central Plane Deflection " $\overline{\mathrm{w}}$ ” at $(\mathrm{a} / 2, \mathrm{~b} / 2)$

Table 1. contains the nondimenionalized deflections for 3-(square), 5-, 7-, and 9layers laminate. The results were normalized using the following terms [2],

$\overline{\mathrm{w}}=\pi^{4} \mathrm{Q} \mathrm{w} / 12 \mathrm{q}_{\mathrm{o}} \mathrm{hS}^{4}$ where $\mathrm{Q}=4 \mathrm{G}_{\mathrm{LT}}+\left[\mathrm{E}_{\mathrm{L}}+\mathrm{E}_{\mathrm{T}}\left(1+2 \mathrm{v}_{\mathrm{TT}}\right)\right] /\left(1-\mathrm{v}_{\mathrm{LT}} \mathrm{v}_{\mathrm{TL}}\right)$ and $\mathrm{S}=\mathrm{a} / \mathrm{h}$

While Table 2. contains results for 3-(rectangular), and 4-layers laminate. The results are normalized using the following formula [1]

$$
\overline{\mathrm{w}}=100 \mathrm{E}_{22} \frac{\mathrm{W}}{\sigma \mathrm{hS}^{4}} ; \sigma=\mathrm{q}_{\text {o }}
$$

The In-Plane Stress

The results of longitudinal stresses $\left(\bar{\sigma}_{11}, \bar{\sigma}_{22}\right.$ and $\left.\bar{\sigma}_{12}\right)$ is shown in Tables 3., 4., and 5. respectively. For the sake of brevity, results of $\bar{\sigma}_{22}$ is shown only for 3-ply rectangular Laminate. All the results are normalized as follow; [1-2]

$$
\bar{\sigma}_{\alpha \beta}=\sigma_{\alpha \beta} / q_{o} S^{2}
$$

The Transverse Normal Stress

In Table 6., the present results of $\bar{\sigma}_{33}\left(a / 2, b / 2, \bar{X}_{3}\right)$ for 3-ply square Laminate are compared with the elasticity (exact) results obtained by Senthil and Batra [4]. The results are normalized as follow[4]

$$
\bar{\sigma}_{33}=\sigma_{33} / q_{o}
$$

The Transverse Shear Stress

Tables7. and 8. give the nondimenionalized results of $\bar{\sigma}_{13}(0, \mathrm{~b} / 2,0)$ and $\bar{\sigma}_{23}(\mathrm{a} / 2,0,0)$ respectively. The following quantity is used for normalization; [1-2]

$$
\bar{\sigma}_{\alpha 3}=\sigma_{\alpha 3} / \sigma S ; \sigma=q_{o}
$$

The Longitudinal Displacement

The results for the in-plane displacement are obtained using [3] 


$$
\mathrm{u}_{1}=\psi_{1} * \mathrm{X}_{3}
$$

Table 9. lists the elasticity results of $\overline{\mathrm{u}}_{1}(\mathrm{a} / 4, \mathrm{~b} / 2, \pm \mathrm{h} / 2)$ obtained by Senthil and Batra [4] as a comparison with the present results. The results are normalized by [4];

$$
\overline{\mathrm{u}}_{1}=\frac{100 \mathrm{E}_{\mathrm{T}}}{\mathrm{q}_{\mathrm{o}} \mathrm{S}^{3}} \overline{\mathrm{X}}_{3} \psi_{1}
$$

\section{CONCLUSION}

A simplified approach, stress-based, was examined by the authors for laminated plate in cylindrical bending[3] has been extended for simply supported cross-ply symmetric laminates. Average stiffness moduli are considered to characterize the laminate properties, ESL. In order to verify the accuracy of the presented approach, a square plate with 3-, 7-, 5- and 9-layers and a rectangular plate with 3-layers orthotropic laminates of equal thickness has been examined. Aspect ratios (span-tothickness)of 2, 4(thick), 10, 20(intermediate) and 50, 100(thin) have been considered. Numerical results have been compared with the elasticity (exact) solutions and other models (CPT, FSDT, HSDT, LWM, 3-D FE) as well and the following observations are made for

1. The present approach estimates the central deflection very well compared to the elasticity solutions, ES, almost for all the considered aspect ratios,

2. For the transverse shear stress, the present approach is in a good agreement with the elasticity solutions especially for intermediate aspect ratios and yields better results than both the FSDT and HSDT of Reddy [8],

3. The present approach estimates both the transverse normal stresses and the inplane displacement, at the surface, very good as compared to the 3-D elasticity (exact) solutions by Senthil and Batra [4],

4. The present approach is consistency in the sense that it yields results with the same level of accuracy for all the multi-layered laminate considered,

5. The assumption of continuity of the longitudinal stress at the laminate interfaces which violates the continuity of the displacements causes significant differences with the elasticity solutions for the thick and intermediate laminate. However, the present approach compares fairly good for aspect ratio greater than 20 ,

\section{ACKNOWLEDGMENT}

The MathCad program was provided and sat up by LtCol Dr Amgad. Also, his technical advises for using the program are acknowledged.

\section{REFERENCES}

1. Pagano, N.J., "Exact Solution for Bidirectional Composites and Sandwich Plates," J Composite Materials, Vol. 4, 20-34, (1970). 
2. Pagano, N.J. \& S.J. Hatfield "Elastic Behavior of Multilayered Bidirectional Composites", AIAA J., Vol.10, No. 7, 931- 933, 1972.

3. Tmerek, M.T. and El-Soaaly, E.E., "Simplified Variational Approach for Analysis of Thick Laminated Orthotropic Plates:1- Cylindrical Bending." Paper presented to the ASAT-12.

4. Senthil S. Vel and R.C. Batra, "Analytical Solution for Rectangular Thick Laminated Plates Subjected to Arbitrary Boundary Conditions," AIAA J, Vol. 37, No. 11, Nov (1999).

5. Y. W. Kwon and J. E. Akin, "Analysis of Layered Composite Plates Using A Higher Order Deformation Theory," Computers and Structures 27, 619-623 (1987).

6. Mawenya, A.S. and Davies, J.D., "Finite Element Analysis of Multilayer Plates," Int. J. Num. in Eng., Vol.8, 215-225,1974.

7. Owen, D.R.J. and Li, Z.H., "A Refined Analysis of Laminated Plates by Finite Element Displacement Method- I. Fundamentals and Static Analysis," Computers and Structures 26, 907-914 (1987).

8. Reddy, J.N., "A Simple Higher Order Theory for Laminated Composite Plates," ASME J Applied Mech, Vol 51, 12, pp 745 - 752, 1984. 


\section{APPENDIX}

\section{Equations of The Generalized Displacements}

$\Psi^{\prime}=\frac{6}{5} \cdot \mathrm{q} \cdot \frac{\mathrm{b}^{2} \cdot \mathrm{a} \cdot\left(\pi^{4} \cdot \mathrm{b}^{4} \cdot \mathrm{h}^{4} \cdot \mathrm{B} 1 \cdot \mathrm{Q} 66 \cdot \mathrm{d} 1+\pi^{4} \cdot \mathrm{h}^{4} \cdot \mathrm{B} 1 \cdot \mathrm{b}^{2} \cdot \mathrm{Q} 22 \cdot \mathrm{a}^{2} \cdot \mathrm{d} 1+12 \cdot \pi^{2} \cdot \mathrm{b}^{4} \cdot \mathrm{h}^{2} \cdot \mathrm{B} 1 \cdot \mathrm{a}^{2} \cdot \mathrm{d} 2 \cdot \mathrm{d} 1+\pi^{4} \cdot \mathrm{h}^{4} \cdot \mathrm{B} 1 \cdot \mathrm{a}^{2}\right.}{\pi^{3} \cdot \mathrm{h}^{3} \cdot\left(2 \cdot \pi^{2} \cdot \mathrm{h}^{2} \cdot \mathrm{a}^{2} \cdot \mathrm{Q} 66 \cdot \mathrm{Q} 12 \cdot \mathrm{b}^{4} \cdot \mathrm{d} 1-\pi^{2} \cdot \mathrm{h}^{2} \cdot \mathrm{Q} 66 \cdot \mathrm{Q} 11 \cdot \mathrm{b}^{6} \cdot \mathrm{d} 1-12 \cdot \mathrm{Q} 11 \cdot \mathrm{d} 2 \cdot \mathrm{b}^{6} \cdot \mathrm{a}^{2} \cdot \mathrm{d} 1+\pi^{2}\right.}$

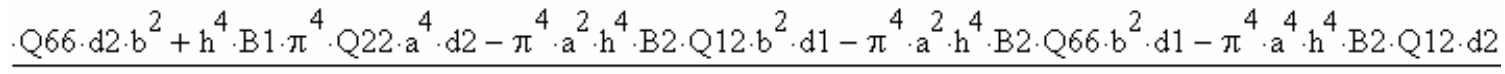
$\cdot b^{4} \cdot h^{2} \cdot Q 12^{2} \cdot a^{2} \cdot d 1-\pi^{2} \cdot b^{4} \cdot h^{2} \cdot Q 22 \cdot Q 11 \cdot a^{2} \cdot d 1-48 \cdot Q 66 \cdot a^{4} \cdot b^{4} \cdot d 2 \cdot d 1-24 \cdot a^{4} \cdot Q 12 \cdot b^{4} \cdot d 2 \cdot d 1+2 \cdot \pi^{2} \cdot a^{4} \cdot h^{2} \cdot Q 12 \cdot$ $+12 \cdot \pi^{2} \cdot a^{4} \cdot h^{2} \cdot B 2 \cdot d 2 \cdot b^{2} \cdot d 1-\pi^{4} \cdot a^{4} \cdot h^{4} \cdot B 2 \cdot Q 66 \cdot d 2-10 \cdot a^{2} \cdot \pi^{2} \cdot h^{2} \cdot Q 66 \cdot b^{4} \cdot d 1+10 \cdot a^{4} \cdot h^{2} \cdot \pi^{2} \cdot Q 12 \cdot d 2 \cdot b^{2}-120 \cdot a^{4}$ $066 \cdot d 2 \cdot b^{2}-\pi^{2} \cdot b^{2} \cdot h^{2} \cdot 022 \cdot 066 \cdot a^{4} \cdot d 1-\pi^{2} \cdot h^{2} \cdot 011 \cdot 066 \cdot d 2 \cdot a^{2} \cdot b^{4}-\pi^{2} \cdot h^{2} \cdot 011 \cdot a^{4} \cdot b^{2} \cdot O 22 \cdot d 2+\pi^{2} \cdot a^{4} \cdot h^{2} \cdot 012^{2}$ $\frac{\left.b^{4} \cdot d 2 \cdot d 1-10 \cdot a^{4} \cdot \pi^{2} \cdot b^{2} \cdot h^{2} \cdot Q 22 \cdot d 1+10 \cdot a^{4} \cdot h^{2} \cdot Q 66 \cdot \pi^{2} \cdot d 2 \cdot b^{2}\right)}{}$

$\left.\cdot d 2 \cdot b^{2}-12 \cdot b^{2} \cdot Q 22 \cdot a^{6} \cdot d 2 \cdot d 1-\pi^{2} \cdot h^{2} \cdot Q 66 \cdot Q 22 \cdot a^{6} \cdot d 2\right)$

$\Psi 2=\frac{6}{5} \cdot \mathrm{a} \cdot \frac{\mathrm{a}^{2} \cdot \mathrm{b} \cdot\left(\pi^{4} \cdot \mathrm{h}^{4} \cdot \mathrm{B} 1 \cdot \mathrm{b}^{4} \cdot \mathrm{Q} 66 \cdot \mathrm{d} 1+\pi^{4} \cdot \mathrm{h}^{4} \cdot \mathrm{B} 1 \cdot \mathrm{Q} 12 \cdot \mathrm{b}^{4} \cdot \mathrm{d} 1-12 \cdot \pi^{2} \cdot \mathrm{h}^{2} \cdot \mathrm{B} 1 \cdot \mathrm{a}^{2} \cdot \mathrm{d} 2 \cdot \mathrm{b}^{4} \cdot \mathrm{d} 1+\pi^{4} \cdot \mathrm{h}^{4} \cdot \mathrm{B} 1 \cdot \mathrm{b}^{2} \cdot \mathrm{Q} 66\right.}{\pi^{3} \cdot \mathrm{h}^{3} \cdot\left[(-2) \cdot \pi^{2} \cdot \mathrm{h}^{2} \cdot \mathrm{a}^{2} \cdot \mathrm{Q} 66 \cdot \mathrm{Q} 12 \cdot \mathrm{b}^{4} \cdot \mathrm{d} 1+\pi^{2} \cdot \mathrm{h}^{2} \cdot \mathrm{Q} 66 \cdot \mathrm{Q} 11 \cdot \mathrm{b}^{6} \cdot \mathrm{d} 1+12 \cdot \mathrm{Q} 11 \cdot \mathrm{d} 2 \cdot \mathrm{b}^{6} \cdot \mathrm{a}^{2} \cdot \mathrm{d} 1-\pi^{2} \cdot\right.}$ $\frac{a^{2} \cdot d 2+\pi^{4} \cdot h^{4} \cdot B 1 \cdot a^{2} \cdot Q 12 \cdot d 2 \cdot b^{2}-\pi^{4} \cdot h^{4} \cdot B 2 \cdot Q 11 \cdot b^{4} \cdot d 1-\pi^{4} \cdot a^{2} \cdot h^{4} \cdot B 2 \cdot b^{2} \cdot Q 66 \cdot d 1-\pi^{4} \cdot a^{2} \cdot h^{4} \cdot B 2 \cdot Q 11 \cdot d 2 \cdot b^{2}-}{b^{4} \cdot h^{2} \cdot Q 12^{2} \cdot a^{2} \cdot d 1+\pi^{2} \cdot b^{4} \cdot h^{2} \cdot Q 22 \cdot Q 11 \cdot a^{2} \cdot d 1+48 \cdot Q 66 \cdot a^{4} \cdot d 2 \cdot b^{4} \cdot d 1+24 \cdot a^{4} \cdot Q 12 \cdot d 2 \cdot b^{4} \cdot d 1-2 \cdot \pi^{2} \cdot a^{4} \cdot h^{2} \cdot Q 12}$

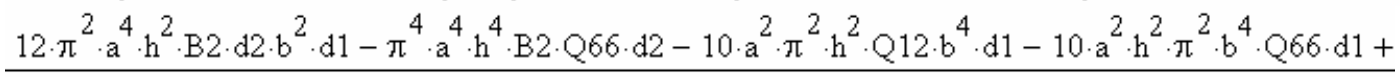
$\mathrm{Q} 66 \cdot \mathrm{d} 2 \cdot \mathrm{b}^{2}+\pi^{2} \cdot \mathrm{b}^{2} \cdot \mathrm{h}^{2} \cdot \mathrm{Q} 22 \cdot \mathrm{Q} 66 \cdot \mathrm{a}^{4} \cdot \mathrm{d} 1+\pi^{2} \cdot \mathrm{h}^{2} \cdot \mathrm{Q} 11 \cdot \mathrm{Q} 66 \cdot \mathrm{d} 2 \mathrm{a}^{2} \cdot \mathrm{b}^{4}+\pi^{2} \cdot \mathrm{h}^{2} \cdot \mathrm{Q} 11 \cdot \mathrm{a}^{4} \cdot \mathrm{b}^{2} \cdot \mathrm{Q} 22 \cdot \mathrm{d} 2-\pi^{2} \cdot \mathrm{a}^{4}$ $\left.120 \cdot a^{4} \cdot d 2 \cdot b^{4} \cdot d 1+10 \cdot a^{2} \cdot \pi^{2} \cdot h^{2} \cdot Q 11 \cdot d 2 \cdot b^{4}+10 \cdot a^{4} \cdot \pi^{2} \cdot h^{2} \cdot b^{2} \cdot Q 66 \cdot d 2\right)$ $\left.\mathrm{h}^{2} \cdot \mathrm{Q} 12^{2} \cdot \mathrm{b}^{2} \cdot \mathrm{d} 2+12 \cdot \mathrm{b}^{2} \cdot \mathrm{Q} 22 \cdot \mathrm{a}^{6} \cdot \mathrm{d} 2 \cdot \mathrm{d} 1+\pi^{2} \cdot \mathrm{h}^{2} \cdot \mathrm{Q} 66 \cdot \mathrm{Q} 22 \cdot \mathrm{a}^{6} \cdot \mathrm{d} 2\right]$

$\mathrm{W}=\frac{1}{5} \cdot \mathrm{q} \cdot \mathrm{a}^{2} \cdot \mathrm{b}^{2} \cdot \underline{\left[(-6) \cdot \pi^{4} \cdot \mathrm{h}^{4} \cdot \mathrm{B} 1 \cdot \mathrm{Q} 66 \cdot \mathrm{b}^{4} \cdot \mathrm{d} 1-72 \cdot \pi^{2} \cdot \mathrm{h}^{2} \cdot \mathrm{B} 1 \cdot \mathrm{b}^{4} \cdot \mathrm{a}^{2} \cdot \mathrm{d} 2 \cdot \mathrm{d} 1-6 \cdot \pi^{4} \cdot \mathrm{h}^{4} \cdot \mathrm{B} 1 \cdot \mathrm{Q} 22 \cdot \mathrm{a}^{2} \cdot \mathrm{b}^{2}\right.}$

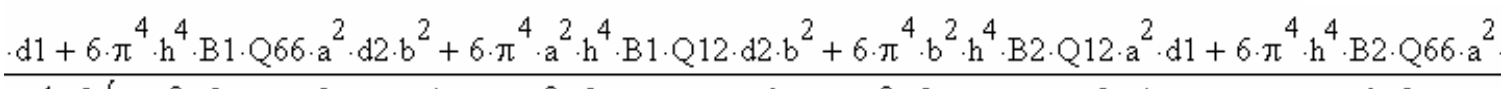
$\pi^{4} \cdot h^{3} \cdot\left(2 \cdot \pi^{2} \cdot h^{2} \cdot Q 66 \cdot a^{2} \cdot Q 12 \cdot b^{4} \cdot d 1-\pi^{2} \cdot h^{2} \cdot Q 66 \cdot Q 11 \cdot b^{6} \cdot d 1-\pi^{2} \cdot h^{2} \cdot Q 22 \cdot Q 11 \cdot a^{2} \cdot b^{4} \cdot d 1-12 \cdot Q 11 \cdot b^{6} \cdot a^{2} \cdot d 2 \cdot d 1\right.$ $\frac{b^{2} \cdot d 1-6 \cdot \pi^{4} \cdot h^{4} \cdot B 2 \cdot Q 11 \cdot a^{2} \cdot d 2 \cdot b^{2}-6 \cdot \pi^{4} \cdot h^{4} \cdot B 2 \cdot Q 66 \cdot a^{4} \cdot d 2-72 \cdot \pi^{2} \cdot h^{2} \cdot B 2 \cdot a^{4} \cdot d 2 \cdot b^{2} \cdot d 1-10 \cdot \pi^{4} \cdot h^{4} \cdot Q 66 \cdot a^{2}}{+\pi^{2} \cdot b^{4} \cdot h^{2} \cdot Q 12^{2} \cdot a^{2} \cdot d 1-48 \cdot Q 66 \cdot a^{4} \cdot d 2 \cdot b^{4} \cdot d 1-24 \cdot a^{4} \cdot Q 12 \cdot d 2 \cdot b^{4} \cdot d 1-\pi^{2} \cdot h^{2} \cdot Q 22 \cdot Q 66 \cdot a^{4} \cdot b^{2} \cdot d 1-\pi^{2} \cdot h^{2} \cdot 1}$ $\frac{\mathrm{Q} 12 \cdot \mathrm{b}^{2}+5 \cdot \pi^{4} \cdot \mathrm{h}^{4} \cdot \mathrm{Q} 66 \cdot \mathrm{Q} 11 \cdot \mathrm{b}^{4}+60 \cdot \pi^{2} \cdot \mathrm{h}^{2} \cdot \mathrm{Q} 11 \cdot \mathrm{b}^{4} \cdot \mathrm{a}^{2} \cdot \mathrm{d} 2+60 \cdot \pi^{2} \cdot \mathrm{h}^{2} \cdot \mathrm{Q} 66 \cdot \mathrm{b}^{4} \cdot \mathrm{a}^{2} \cdot \mathrm{d} 1+5 \cdot \pi^{4} \cdot \mathrm{h}^{4} \cdot \mathrm{Q} 22 \cdot \mathrm{Q} 11 \cdot \mathrm{a}^{2} \cdot}{}$ Q11.Q66 $\mathrm{a}^{2} \cdot \mathrm{d} 2 \cdot \mathrm{b}^{4}+2 \cdot \pi^{2} \cdot \mathrm{a}^{4} \cdot \mathrm{h}^{2} \cdot \mathrm{Q} 12 \cdot \mathrm{Q} 66 \cdot \mathrm{d} 2 \cdot \mathrm{b}^{2}+\mathrm{a}^{4} \cdot \pi^{2} \cdot \mathrm{h}^{2} \cdot \mathrm{Q} 12^{2} \cdot \mathrm{b}^{2} \cdot \mathrm{d} 2-\pi^{2} \cdot \mathrm{h}^{2} \cdot \mathrm{Q} 11 \cdot \mathrm{Q} 22 \cdot \mathrm{a}^{4} \cdot \mathrm{d} 2 \cdot \mathrm{b}^{2}-\pi^{2} \cdot \mathrm{h}^{2}$ $b^{2}-5 \cdot \pi^{4} \cdot a^{2} \cdot b^{2} \cdot h^{4} \cdot Q 12^{2}+720 \cdot a^{4} \cdot b^{4} \cdot d 2 \cdot d 1+60 \cdot \pi^{2} \cdot h^{2} \cdot Q 66 \cdot a^{4} \cdot d 2 \cdot b^{2}+5 \cdot \pi^{4} \cdot h^{4} \cdot Q 22 \cdot Q 66 \cdot a^{4}$ $\left.\cdot \mathrm{Q} 66 \cdot \mathrm{Q} 22 \cdot \mathrm{a}^{6} \cdot \mathrm{d} 2-12 \cdot \mathrm{Q} 22 \cdot \mathrm{a}^{6} \cdot \mathrm{d} 2 \cdot \mathrm{b}^{2} \cdot \mathrm{d} 1\right)$

$$
\left.+60 \cdot \pi^{2} \cdot h^{2} \cdot Q 22 \cdot a^{4} \cdot b^{2} \cdot d 1\right]
$$




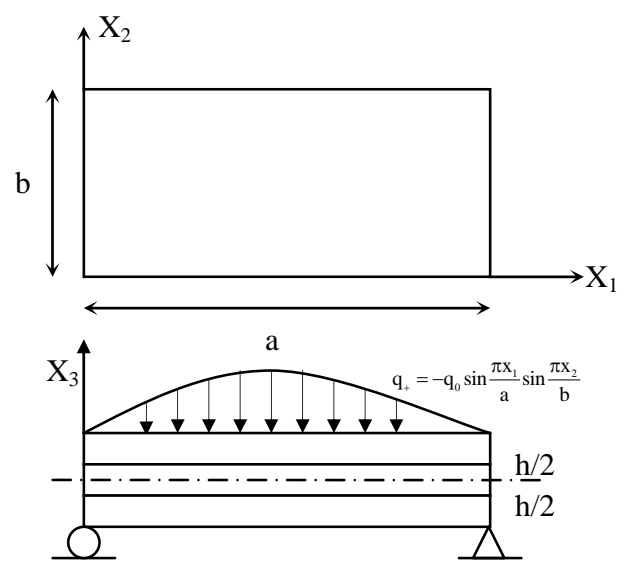

Fig. 1 Orthotropic laminated simply supported plate.

Table 1. Central deflections “ $\overline{\mathrm{w}}$ " at $(\mathrm{a} / 2, \mathrm{~b} / 2)$, normalized as Pagano and Hatfield[2]

\begin{tabular}{|c|c|c|c|c|c|c|c|c|c|}
\hline \multicolumn{5}{|c|}{ 3-ply square Laminate } & \multicolumn{5}{|c|}{ 5-ply Laminate } \\
\hline $\mathrm{S}$ & $\mathrm{ES}^{1}$ & Present & {$[5]$} & [6] & $\mathrm{ES}^{1}$ & Present & [6] & [7] & [8] \\
\hline 2 & 11.767 & 11.159 & 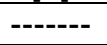 & 14.731 & 12.278 & 11.197 & 13.862 & ----- & ----- \\
\hline 4 & 4.491 & 3.57 & ------ & 4.934 & 4.291 & 3.571 & 4.331 & ----- & 4.244 \\
\hline 5 & ----- & 2.657 & 3.621 & ----- & $\begin{array}{ll}---- \\
\end{array}$ & $\begin{array}{ll}---- \\
\end{array}$ & $\begin{array}{ll}---- \\
\end{array}$ & $\begin{array}{l}---- \\
\end{array}$ & ----- \\
\hline 10 & 1.709 & 1.435 & 1.788 & 1.758 & 1.57 & 1.433 & 1.57 & ----- & 1.551 \\
\hline 20 & 1.189 & 1.128 & ------ & 1.196 & 1.145 & 1.127 & 1.142 & 1.232 & 1.135 \\
\hline 50 & 1.031 & 1.041 & ----- & 1.026 & 1.023 & 1.041 & 1.017 & 1.041 & 1.015 \\
\hline \multirow[t]{2}{*}{100} & 1.008 & 1.029 & ----- & 1.002 & 1.006 & 1.029 & 0.999 & 1.013 & 0.998 \\
\hline & CPT & 1 & & & СРT & 1 & & & \\
\hline \multicolumn{10}{|c|}{${ }^{1}$ ES, Elasticity (Exact); [2] } \\
\hline \multicolumn{5}{|c|}{ 7-ply Laminate } & \multicolumn{5}{|c|}{ 9-ply Laminate } \\
\hline $\mathrm{S}$ & $E^{1}$ & Present & & & $\mathrm{ES}^{1}$ & Present & [8] & & \\
\hline 2 & 12.342 & 11.194 & & & 12.288 & 11.193 & ----- & & \\
\hline 4 & 4.153 & 3.569 & & & 4.079 & 3.568 & 4.088 & & \\
\hline 10 & 1.529 & 1.432 & & & 1.512 & 1.432 & 1.505 & & \\
\hline 20 & 1.133 & 1.126 & & & 1.129 & 1.126 & 1.123 & & \\
\hline 50 & 1.021 & 1.041 & & & 1.021 & 1.041 & 1.016 & & \\
\hline \multirow[t]{2}{*}{100} & 1.005 & 1.029 & & & 1.005 & 1.029 & 1.000 & & \\
\hline & CPT & 1 & & & CPT & 1 & & & \\
\hline
\end{tabular}

Table 2. Central deflections " $\overline{\mathrm{w}}$ ” at $(\mathrm{a} / 2, \mathrm{~b} / 2)$, normalized after Pagano [1]

\begin{tabular}{|c|c|c|c|c|c|c|}
\hline \multicolumn{7}{|c|}{ 3-ply rectangular Laminate } \\
\hline $\mathrm{S}$ & ES & Present & {$[8]^{2}$} & {$[8]^{3}$} & [5] & [6] \\
\hline 2 & 8.17 & 7.797 & ---- & ---- & ---- & ---- \\
\hline 4 & 2.82 & 2.513 & 2.64 & 2.36 & ---- & ---- \\
\hline 10 & 0.92 & 0.999 & 0.86 & 0.8 & 0.93 & ----- \\
\hline 20 & 0.61 & 0.78 & 0.59 & 0.58 & 0.61 & 0.67 \\
\hline 50 & 0.52 & 0.719 & ----- & ----- & 0.52 & 0.53 \\
\hline 100 & 0.51 & 0.71 & 0.51 & 0.51 & 0.51 & 0.51 \\
\hline & СРТ & 0.503 & & & & \\
\hline
\end{tabular}

\begin{tabular}{|c|r|r|r|r|}
\hline \multicolumn{6}{|c|}{ 4-ply Laminate } \\
\hline ES $^{1}$ & present & \multicolumn{1}{c|}{$[8]^{2}$} & \multicolumn{1}{c|}{$[8]^{3}$} & \multicolumn{1}{c|}{$[7]$} \\
\hline 1.954 & 1.502 & 1.89 & 1.71 & 1.87 \\
\hline 0.743 & 0.603 & 0.72 & 0.66 & 0.71 \\
\hline 0.517 & 0.474 & 0.51 & 0.49 & 0.5 \\
\hline 0.434 & 0.438 & ---- & ---- & 0.43 \\
\hline 0.4385 & 0.433 & 0.43 & 0.43 & 0.42 \\
\hline
\end{tabular}


Table 3. Normalized longitudinal stress; $\bar{\sigma}_{11}$ at $(\mathrm{a} / 2, \mathrm{~b} / 2, \mathrm{~h} / 2)$

\begin{tabular}{|c|c|c|c|c|c|c|c|c|c|c|}
\hline \multicolumn{5}{|c|}{ 3-ply square Laminate } & \multicolumn{6}{|c|}{ 3-ply rectangular Laminate } \\
\hline S & present & \multicolumn{2}{|c|}{ ES[2] } & $E S[1]$ & \multicolumn{2}{|c|}{ present } & \multicolumn{2}{|c|}{\begin{tabular}{l|l} 
ES &
\end{tabular}} & {$\left[\begin{array}{l}{[8]^{3}} \\
\end{array}\right.$} & [6] \\
\hline 2 & 0.331 & \multicolumn{2}{|c|}{1.388} & ------ & \multicolumn{2}{|c|}{0.566} & \multicolumn{2}{|c|}{2.13} & --- & --- \\
\hline 4 & 0.338 & \multicolumn{2}{|c|}{0.72} & ------ & \multicolumn{2}{|c|}{0.582} & 1.14 & 14 & 0.613 & ---- \\
\hline 5 & 0.343 & \multicolumn{2}{|c|}{------ } & 0.718 & \multicolumn{2}{|c|}{------ } & ----- & $--\cdot$ & ----- & --- \\
\hline 10 & 0.357 & \multicolumn{2}{|c|}{0.559} & 0.591 & \multicolumn{2}{|c|}{0.593} & 0.726 & & 0.622 & --- \\
\hline 20 & 0.365 & 0.5 & & ----- & & 95 & 0. & & 0.623 & 0.651 \\
\hline 50 & 0.367 & 0.5 & & ----- & & 95 & 0.6 & 28 & --- & 0.64 \\
\hline 100 & 0.368 & 0.5 & & ------ & & 95 & 0.6 & & 0.623 & 0.638 \\
\hline & CPT & 0.53 & & & & & CP1 & 0.6 & & \\
\hline & & & & & & & & ${ }^{2} \mathrm{HSDT}^{3}$ & DT & \\
\hline & & -ply Lamir & ate & & & & & 5-ply Lar & רate & \\
\hline $\mathrm{S}$ & $\mathrm{ES}^{1}$ & present & {$[8]^{2}$} & {$[8]^{3}$} & $\mathrm{~S}$ & pre & sent & $E S[2]$ & [6] & \\
\hline 4 & 0.72 & 0.28 & 0.67 & 0.41 & 2 & & 313 & 1.332 & ----- & \\
\hline 10 & 0.56 & 0.28 & 0.55 & 0.5 & 1 & & 17 & 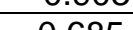 & 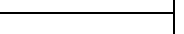 & \\
\hline 20 & 0.54 & 0.28 & 0.54 & 0.53 & & & & -0.651 & & \\
\hline 100 & 0.54 & 0.28 & 0.54 & 0.54 & 5 & & 19 & -------- & --- & \\
\hline${ }^{1}$ result & quoted by & $y[8]$ & ${ }^{2} \mathrm{HSI}$ & DT ${ }^{3} \mathrm{FSDT}$ & 10 & & 327 & 0.545 & --- & \\
\hline & & & & & 20 & & 332 & 0.539 & 0.548 & \\
\hline & & & & & 50 & & 34 & 0.539 & 0.55 & \\
\hline & & & & & 100 & & 34 & 0.539 & 0.551 & \\
\hline & & & & & & & DТ & 0.539 & & \\
\hline & & -ply Lamir & & & & & & 9-ply Lar & nate & \\
\hline $\mathrm{s}$ & present & ES[2] & & & S & & sent & $\mathrm{ES}[2]$ & & \\
\hline 2 & 0.306 & $\begin{array}{l}1.284 \\
-0.88\end{array}$ & & & 2 & & 01 & $\begin{array}{c}1.26 \\
-0.866\end{array}$ & & \\
\hline 4 & 0.307 & $\begin{array}{c}0.679 \\
-0.645\end{array}$ & & & 4 & & 02 & $\begin{array}{c}0.684 \\
-0.649\end{array}$ & & \\
\hline 10 & 0.314 & 0.548 & & & 10 & & 07 & 0.551 & & \\
\hline 20 & 0.318 & 0.539 & & & 20 & & 31 & 0.541 & & \\
\hline 50 & 0.319 & 0.539 & & & 50 & & 11 & 0.539 & & \\
\hline 100 & 0.319 & 0.539 & & & 100 & & 11 & 0.539 & & \\
\hline & СРТ & 0.539 & & & & & गT & 0.539 & & \\
\hline
\end{tabular}


Table 4. Normalized longitudinal stress; $\bar{\sigma}_{22}$ at $(\mathrm{a} / 2, \mathrm{~b} / 2, \mathrm{~h} / 6)$

\begin{tabular}{|c|c|c|c|c|}
\hline $\mathrm{S}$ & ES[1] & Present & {$[8]^{2}$} & {$[8]^{3}$} \\
\hline 2 & 0.23 & 0.066 & ----- & ----- \\
\hline 4 & 0.119 & 0.036 & 0.103 & 0.093 \\
\hline 10 & 0.044 & 0.019 & 0.04 & 0.038 \\
\hline 20 & 0.0299 & 0.016 & 0.0289 & 0.0283 \\
\hline \multirow[t]{2}{*}{100} & 0.0253 & 0.015 & 0.0253 & 0.0253 \\
\hline & CPT & 0.0252 & ${ }^{2} \mathrm{HSDT}$ & FSDT \\
\hline
\end{tabular}

Table 5. Normalized longitudinal stress; $\bar{\sigma}_{12}$ at $(0,0, \mathrm{~h} / 2)$

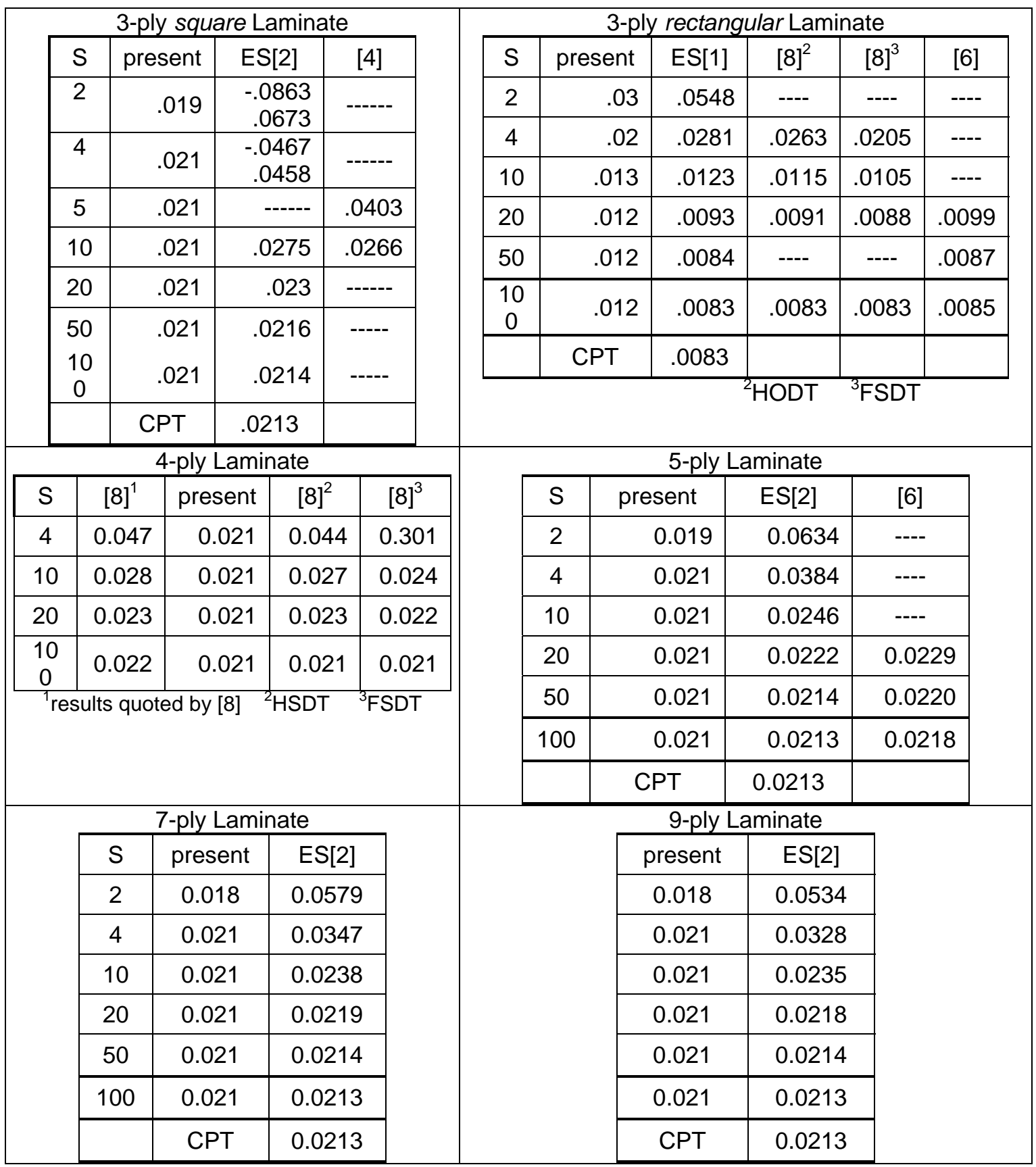


Table 6. Nondimenionalized transverse normal stress; $\bar{\sigma}_{33}$ at $\left(\mathrm{a} / 2, \mathrm{~b} / 2, \overline{\mathrm{X}}_{3}\right)$

\begin{tabular}{|c|c|r|r|}
\hline$\overline{\mathrm{X}}_{3}$ & $\mathrm{~S}$ & Present & \multicolumn{1}{c|}{$[4]$} \\
\hline $\mathrm{h}$ & 5 & 0.741 & 0.726 \\
\cline { 2 - 4 } 6 & 10 & 0.741 & 0.74 \\
\cline { 3 - 4 } 0 & 5 & 0.5 & 0.496 \\
\cline { 3 - 5 } & 10 & 0.5 & 0.5 \\
\hline
\end{tabular}

Table 7. Normalized transverse shear stress ; $\bar{\sigma}_{13}$ at $(0, \mathrm{~b} / 2,0)$

\begin{tabular}{|c|c|c|c|c|c|c|c|c|c|c|}
\hline \multicolumn{6}{|c|}{ 3-ply square Laminate } & \multicolumn{5}{|c|}{ 3-ply rectangular Laminate } \\
\hline$S$ & present & ES[2] & \multicolumn{2}{|c|}{$[4]$} & & S & present & $\mathrm{ES}[1]$ & {$[8]^{2}$} & {$[8]^{3}$} \\
\hline 2 & 0.275 & 0.153 & \multicolumn{2}{|c|}{------ } & & 2 & 0.452 & 0.257 & ---- & ---- \\
\hline 4 & 0.282 & 0.219 & \multicolumn{2}{|c|}{$\begin{array}{l}----- \\
---\end{array}$} & & 4 & 0.463 & 0.351 & 0.273 & 0.1879 \\
\hline 5 & 0.286 & ------ & \multicolumn{2}{|c|}{0.2653} & & 10 & 0.469 & 0.42 & 0.286 & 0.1894 \\
\hline 10 & 0.297 & 0.301 & \multicolumn{2}{|c|}{0.3301} & & 20 & 0.47 & 0.434 & 0.288 & 0.1896 \\
\hline 20 & 0.303 & 0.328 & \multicolumn{2}{|c|}{------ } & & 50 & 0.471 & 0.439 & ----- & ----- \\
\hline 50 & 0.305 & 0.337 & \multicolumn{2}{|c|}{------ } & & 100 & 0.471 & 0.439 & 0.289 & 0.1897 \\
\hline \multirow[t]{2}{*}{100} & 0.306 & 0.339 & \multicolumn{2}{|c|}{----- } & & & $\mathrm{CPT}$ & 0.44 & \multirow[t]{2}{*}{${ }^{2} \mathrm{HSDT}$} & \multirow[t]{2}{*}{${ }^{3} \mathrm{FSDT}$} \\
\hline & CPT & 0.339 & & & & & & & & \\
\hline \multicolumn{6}{|c|}{ 4-ply Laminate } & \multicolumn{4}{|c|}{ 5-ply Laminate } & \\
\hline$S$ & $\mathrm{ES}^{1}$ & Present & {$[8]^{2}$} & {$[8]^{3}$} & [7] & $S$ & Present & $E S[2]$ & [7] & \\
\hline 4 & 0.291 & 0.239 & 0.206 & 0.14 & 0.229 & 2 & 0.261 & 0.227 & ---- & \\
\hline 10 & 0.301 & 0.239 & 0.264 & 0.167 & 0.315 & 4 & 0.265 & 0.238 & 0.250 & \\
\hline 20 & 0.328 & 0.239 & 0.283 & 0.175 & 0.343 & 10 & 0.274 & 0.258 & 0.278 & \\
\hline 100 & 0.337 & 0.239 & 0.29 & 0.178 & 0.355 & 20 & 0.277 & 0.268 & 0.290 & \\
\hline & results quc & loted by $[8]$ & ] ${ }^{2} \mathrm{HS}$ & ${ }^{3} \mathrm{~F}$ & DT & 50 & 0.279 & 0.271 & 0.298 & \\
\hline & & & & & & 100 & 0.279 & 0.272 & 0.308 & \\
\hline & & & & & & & СРТ & 0.272 & & \\
\hline & & 7-ply La & aminat & & & & & ly Lamina & & \\
\hline$S$ & present & $\mathrm{ES}[2$ & & & & S & present & ES[2] & [7] & \\
\hline 2 & 0.25 & 0.1 & 178 & & & 2 & 0.251 & 0.204 & --- & \\
\hline 4 & 0.25 & 0.2 & 219 & & & 4 & 0.253 & 0.223 & 0.229 & \\
\hline 10 & 0.26 & 0.2 & 255 & & & 10 & 0.258 & 0.247 & 0.256 & \\
\hline 20 & 0.26 & 0.2 & 267 & & & 20 & 0.26 & 0.255 & 0.265 & \\
\hline 50 & 0.26 & 0.2 & 271 & & & 50 & 0.261 & 0.258 & 0.269 & \\
\hline 100 & 0.26 & 0.2 & 272 & & & 100 & 0.261 & 0.259 & 0.270 & \\
\hline & СРТ & 0.27 & & & & & СРТ & 0.259 & & \\
\hline
\end{tabular}


Table 8. Normalized transverse shear stress; $\bar{\sigma}_{23}$ at $(\mathrm{a} / 2,0,0)$

\begin{tabular}{|c|c|c|c|c|c|c|c|c|c|c|}
\hline \multicolumn{6}{|c|}{ 3-ply square Laminate } & \multicolumn{5}{|c|}{ 3-ply rectangular Laminate } \\
\hline S & present & ES[2] & [4] & & & $S$ & present & $E S[1]$ & {$[8]^{2}$} & {$[8]^{3}$} \\
\hline 2 & 0.203 & 0.295 & ------ & & & 2 & 0.075 & 0.0668 & ---- & --- \\
\hline 4 & 0.195 & 0.292 & ------ & & & 4 & 0.044 & 0.0334 & 0.0348 & 0.0308 \\
\hline 5 & 0.191 & ----- & 0.1911 & & & 5 & 0.037 & ----- & --- & ---- \\
\hline 10 & 0.18 & 0.196 & 0.1228 & & & 10 & 0.025 & 0.0152 & 0.017 & 0.0159 \\
\hline 20 & 0.174 & 0.156 & ------ & & & 20 & 0.022 & 0.0119 & 0.0139 & 0.0135 \\
\hline 50 & 0.172 & 0.141 & ------ & & & 50 & 0.021 & 0.0110 & ----- & ---- \\
\hline \multirow[t]{2}{*}{100} & 0.172 & 0.139 & ------ & & & 100 & 0.021 & 0.0108 & 0.0129 & 0.0127 \\
\hline & CPT & 0.138 & & & & & CPT & 0.0108 & ${ }^{2} \mathrm{HSDT}$ & ${ }^{3}$ FSDT \\
\hline \multicolumn{6}{|c|}{ 4-ply Laminate $(0 / 90 / 90 / 0) ; b=a$} & \multicolumn{4}{|c|}{ 5-ply Laminate } & \\
\hline $\mathrm{s}$ & $\mathrm{ES}^{1}$ & Present & {$[8]^{2}$} & {$[8]^{3}$} & [7] & $S$ & present & ES[2] & {$[7]$} & \\
\hline 4 & 0.292 & 0.239 & 0.239 & 0.1963 & 0.299 & 2 & 0.217 & $\begin{array}{l}0.186 \\
0.268\end{array}$ & --- & \\
\hline 10 & 0.196 & 0.239 & 0.153 & 0.129 & 0.198 & \multirow[t]{2}{*}{4} & \multirow{2}{*}{0.212} & $\begin{array}{l}0.268 \\
0.229\end{array}$ & \multirow{2}{*}{0.257} & \\
\hline 20 & 0.156 & 0.239 & 0.123 & 0.109 & 0.157 & & & 0.233 & & \\
\hline \multirow[t]{5}{*}{100} & 0.141 & 0.239 & 0.112 & 0.101 & 0.144 & 10 & 0.204 & 0.223 & 0.247 & \\
\hline & \multirow[t]{4}{*}{${ }^{1}$ results } & quoted by [ & \multirow[t]{4}{*}{ 8] ${ }^{2} \mathrm{HSD}$} & \multirow{4}{*}{\multicolumn{2}{|c|}{${ }^{3} \mathrm{FSDT}$}} & 20 & 0.2 & 0.212 & 0.233 & \\
\hline & & & & & & 50 & 0.199 & 0.206 & 0.231 & \\
\hline & & & & & & 100 & 0.199 & 0.205 & 0.229 & \\
\hline & & & & & & & CPT & 0.205 & & \\
\hline \multicolumn{6}{|c|}{ 7-ply Laminate } & \multicolumn{4}{|c|}{ 9-ply Laminate } & \\
\hline$S$ & present & $\mathrm{ES}[2]$ & & & & $\mathrm{S}$ & present & ES[2] & [7] & \\
\hline 2 & 0.223 & 0.238 & & & & 2 & 0.227 & 0.194 & ------ & \\
\hline 4 & 0.22 & 0.236 & & & & \multirow[t]{2}{*}{4} & \multirow{2}{*}{0.224} & 0.223 & \multirow{2}{*}{0.234} & \\
\hline 10 & 0.214 & 0.219 & & & & & & 0.225 & & \\
\hline 20 & 0.211 & 0.21 & & & & 10 & 0.219 & 0.226 & 0.236 & \\
\hline 50 & 0.21 & 0.206 & & & & 20 & 0.217 & 0.221 & 0.231 & \\
\hline \multirow[t]{2}{*}{100} & 0.21 & 0.205 & & & & 50 & 0.217 & 0.219 & 0.229 & \\
\hline & CPT & 0.205 & & & & 100 & 0.216 & 0.219 & 0.230 & \\
\hline & & & & & & & СРT & 0.205 & & \\
\hline
\end{tabular}

Table 9. Normalized in-plane displacement; $\overline{\mathrm{u}}_{1}$ at $(\mathrm{a} / 4, \mathrm{~b} / 2, \pm \mathrm{h} / 2)$

\begin{tabular}{|c|c|c|c|c|c|}
\hline \multicolumn{6}{|c|}{ 3-ply square Laminate } \\
\hline \multicolumn{3}{|c|}{$S=5$} & \multicolumn{3}{|c|}{$\mathrm{S}=10$} \\
\hline & Present & [4] & & Present & [4] \\
\hline $\mathrm{h} / 2$ & 0.6164 & 0.62 & $h / 2$ & 0.6532 & -0.52 \\
\hline$-h / 2$ & 0.6164 & 0.614 & $-\mathrm{h} / 2$ & 0.6532 & 0.522 \\
\hline
\end{tabular}

